\title{
Scintigraphic detection of regional disruption of adrenergic neurons in the heart
}

\begin{abstract}
Experiments were designed to detect regional disruptions of adrenergic neurons in the hearts of llving dogs. The neuron disuption was achleved by the application of phenol to the eplcardlum of the left ventricle. Evidence tor denervation was the reduction in endogenous norepinephrine (NE) concentrations in the myocardium beneath the region of phenol treatment and toward the apex. Radiolabeled mota-iodobenzylguanidine (MIBG) acts as an analog of NE and as such is concentrated in adrenergic nerve terminals. Following phenol application, MIBG labeled with 125I was found, 20 hours after injection, to be distributed within myocardium in patterns comparable to those of NE. However, left stellectomy did not alter the distributions of NE or ${ }^{125}$ I-MIBG in the myocardium and apparently did not disrupt adrenergic innervation. MIBG labeled with ${ }^{123}$ enabled scintigraphic images of heart neurons in the living dog 3 and 20 hours after injection; these images portrayed the regions of adrenergic neuron disruptlon caused by phenol treatment. Concentrations of thallium-201 depicted on scintigraphic image and of triphenyltetrazolium observed on In vitro staining demonstrated no myocardial injury. Thus scintlgraphy with ${ }^{23}$ |-MIBG will display regional adrenergic denervations in the heart. (AM HEART J 1988;116:67.)
\end{abstract}

James C. Sisson, MD, Joseph J. Lynch, PhD, Jon Johnson, BS, Sandford Jaques, Jr., PhD, Dorothy Wu, BSC, Gerald Bolgos, BS, Benedict R. Lucchesi, MD, PhD, and Donald M. Wieland, PhD. Ann Arbor, Mich.

By a coordinated stimulation of myocardial cells, the adrenergic nervous system acts to maintain normal function in the heart. ${ }^{1}$ Disruption of adrenergic innervation to regions of the heart can leave the more apical parts of the myocardium also denervated. ${ }^{2}$ Upon denervation, adrenergic receptor density increases, ${ }^{3}$ and an imbalanced response of the heart to stimulation by circulating norepinephrine (NE) could ensue. Such events may be a cause of arrhythmias, including those causing death. ${ }^{4}$

However, it has been difficult, if not impossible, to detect regional denervations of the heart. For the most part, tests of adrenergic activity have measured, as in the case of concentrations of catecholamines in the plasma, the sum of the function of many components of the system. Even when assays record the rate of secretion of NE from the heart, ${ }^{5}$ differences in function of adrenergic neurons within

From the University of Michigan Medical Center, Departments of Internal Medicine (Nuclear Medicine) and Pharmacology.

Supported by National Institutes of Health grants DK-21477, HL-37586, HL-05806, and HL-35325; and by the Diabetes Training and Research Center grant MDRTC-5P0DK20572-10.

Received for publication Sept. 16, 1987; accepted March 1, 1988.

Reprint requests: James C. Sisson, MD, University of Michigan Medical Center, 1500 E. Medical Center Dr., University Hospital-BIG 412/0028, Ann Arbor, MI 48109-0028. regions of this organ go undetected. Yet focal abnormalities in adrenergic innervation within the heart are regularly caused by myocardial infarction, ${ }^{6}$ and consequently must occur relatively commonly. We therefore sought a method to detect disruptions of adrenergic neurons within regions of the heart.

Meta-iodobenzylguanidine (MIBG) functions as an analog of NE, the adrenergic neurotransmitter. ${ }^{7}$ As such, MIBG concentrates in adrenergic neuron terminals where, when labeled with ${ }^{123} \mathrm{I}$, it can be detected and quantified in the heart by scintigraphy. ${ }^{8}$ Concentrations of radiolabeled MIBG appear to record faithfully the integrity of adrenergic innervation within the many regions of the heart.

Herein we report that concentrations of radiolabeled MIBG will reflect regional disruptions of adrenergic neurons produced by phenol application to the epicardium of the hearts of dogs. These regional disruptions in neuron integrity were portrayed as readily perceived abnormalities on scintigraphic images made with ${ }^{123}$ I-MIBG in the living animal.

\section{METHODS}

Materials. Mongrel dogs (14.3 to $22.0 \mathrm{~kg}$ ) were purchased from a commercial vendor. "Acute" dogs receiving only ${ }^{125}$ I-MIBG for ex vivo experiments were found to be healthy when observed over 5 to 7 days before study. The 
dogs that received ${ }^{129} \mathrm{I}-\mathrm{MIBG}$ for in vivo scintigraphy were "conditioned" for a period of 30 days; these dogs were clinically normal, immunized against diseases, and free of parasites. All dogs received water ad libitum and regular chow.

The dogs were divided into two groups for testing. Twelve animals had phenol treatment to their hearts; seven of these dogs underwent in vivo scintigraphy with ${ }^{123}$ I-MIBG (in five, the phenol was applied to the epicardium of the anterior wall of the left ventricle, and in two application was to the epicardium on the inferior wall). Ten animals had sham treatment of their hearts; five of these dogs were to undergo in vivo scintigraphy with ${ }^{123}$ I-MIBG (no additional controls were deemed necessary for the two dogs in which the area of phenol application was small-see below), but one died, leaving four in the control scintigraphy group.

Syntheses of ${ }^{126}$ I-MIBG and ${ }^{123}$ I-MIBG were carried out as previously described ${ }^{7 \cdot 9}$ to give $21 \mathrm{mCi}$ (777 gigabecquerel $[\mathrm{GBq}]) / \mathrm{mmol}$ and $1.6 \mathrm{mCi}(59 \mathrm{GBq}) / \mathrm{mmol}$, respectively. Less than $2 \%$ of each radiopharmaceutical was in the form of radiolabeled free iodide. Thallous 201 chloride ( $\left.{ }^{201} \mathrm{Tl}\right)$ was purchased from Mallinkrodt Inc., St. Louis, Mo., and E.I. duPont de Nemours and Company, North Billerica, Mass.

Procedures. For the phenol-treatment experiments, dogs were paired and left thoracotomies were carried out as reported before. ${ }^{10}$ In one dog of each pair, phenol was applied to the epicardium of the heart to produce an injury sufficient to disrupt adrenergic neurons in the epicardium. This method is similar to that described by others. $^{2,11}$ With a cotton-tipped applicator, phenol $(60 \%$ in ethanol; International Biotechnologies, Inc., Farmington, Conn.) was applied to the epicardium in strips 0.2 to $0.3 \mathrm{~cm}$ in width. This was done to inscribe a $3 \mathrm{by} 2 \mathrm{~cm}$ rectangle in the basal region that extended on the anterior wall of the left ventricle from directly below the tip of the left atrial appendage downward to a point that was at least $1 \mathrm{~cm}$ above the apex. The left anterior descending artery then bisected this rectangle. In two dogs, the phenolinscribed rectangle was 2 by $2 \mathrm{~cm}$ and was further away from the apex. In three dogs, phenol was applied to the inferior epicardium so that the rectangle was in the basal region and distal to the first major branch of the left circumflex coronary artery. The second (control) dog of each pair received only the thoracotomy, i.e., the heart was sham-treated. All dogs received a combination antibiotic, trimethoprim, $80 \mathrm{mg}$, and sulfadiazine, $400 \mathrm{mg}$ orally, each day during their post-surgical convalescence.

In a separate group of seven dogs, the left stellate ganglion was excised in an attempt to produce another type of regional denervation. This ganglion was isolated, then the intact body of the structure was removed while severing all pre-ganglionic and postganglionic fibers. In a connrats axup of five dogs, the stellectomy was shown to effect a loss of the left ventricular inotropic response to electrical stimulation of the left sympathetic chain distal to the stellate region. A sham-treated dog was included in each experiment.
To establish a relationship between radiolabeled MIBG and endogenous NE, the initial group of 10 dogs in this investigation received, as a test agent, only ${ }^{125} \mathrm{I}-\mathrm{MIBG}$ in an intravenous dose of $0.2 \mathrm{mCi}$. The ${ }^{125} \mathrm{I}$-MIBG was given 5 or 6 days after the thoracotomy; the dogs were killed 20 hours after the injection. The next 11 dogs were studied by scintigraphy while alive. These dogs were given ${ }^{123}$ I-MIBG, $5 \mathrm{mCi}$ intravenously, 5 to 6 days after thoracotomy. Two to 3 hours and 18 to 20 hours later, scintigraphy was performed with the dogs in the supine position under thiamylal anesthesia. Scintigraphic images of the heart, both planar and tomographic, were created with a GE 400AT scintillation camera (GE Medical Systems, Milwaukee, Wis. $)^{8}$ to which was affixed a low-energy collimator. The camera rotated, in 64 stops, through 180 degrees around the anterior chest of the dogs. At each stop, data were acquired for 30 seconds in the earlier ( 2 to 3 hour) images and for 60 seconds in the later (18 to 20 hour) images; the data were collected in a $64 \times 64$ word matrix, stored, and processed in a computer. The tomographic slices were 1 pixel or $6.5 \mathrm{~mm}$ in thickness.

Two days after injection, the ${ }^{123}$ I-MIBG was almost undetectable. Then ${ }^{201} \mathrm{Tl}, 2.5 \mathrm{mCi}$, was injected intravenously, and planar and tomographic images of this radiopharmaceutical were produced either 15 minutes or 2 hours later. The tomographic data of ${ }^{201} \mathrm{Tl}$ were acquired in 25-second stops.

Three days after injection of ${ }^{201} \mathrm{Tl},{ }^{125} \mathrm{I}-\mathrm{MIBG}, 0.2 \mathrm{mCi}$, was given intravenously; killing was done 20 hours later. At this point ${ }^{125}$ I-MIBG was used because the longer half-life of ${ }^{125}$ I (60 days) enabled the radiopharmaceutical to be more easily measured in the subsubsequent analyses. The distributions of ${ }^{123}$ I-MIBG and ${ }^{125}$ I-MIBG should be identical, and therefore the relative concentrations of ${ }^{125}$ I-MIBG in myocardium at 20 hours served to correlate, in ex vivo studies, with the independent evidence of neuronal injury obtained from levels of endogenous myocardial NE (see below).

The dogs were killed by a method that was designed to give minimal stimulus to the adrenergic nervous system. Anesthesia was induced by Dial-Urethane solution (10\% allobarbital, $40 \%$ monoethylurea, $40 \%$ urethane), 0.6 $\mathrm{ml} / \mathrm{kg}$, intravenously. The dogs were then ventilated with room air by means of a Harvard respirator Harvard Apparatus Co., Natick, Mass. After a left thoracotomy, the hearts were excised during a supramaximal stimulation of the right vagus nerve $(40 \mathrm{~Hz}$ train rate, 4 msec pulse duration, 1.5 times threshold voltage) with a Grass S48 stimulator (Grass Instruments, Quincy, Mass.) to produce a sinus arrest.

Transverse slices of the heart of approximately $0.5 \mathrm{~cm}$ thickness were made perpendicular to the apex-base axis and parallel to the atrioventricular groove. Of particular interest was the basal slice, which contained the region of phenol treatment and could be oriented from the positions of the anterior and posterior papillary muscles for division into 12 reproducible radial segments (Fig. 1). The segments of this basal slice served as a reference for determining the effects of phenol treatment. Transmural sam- 

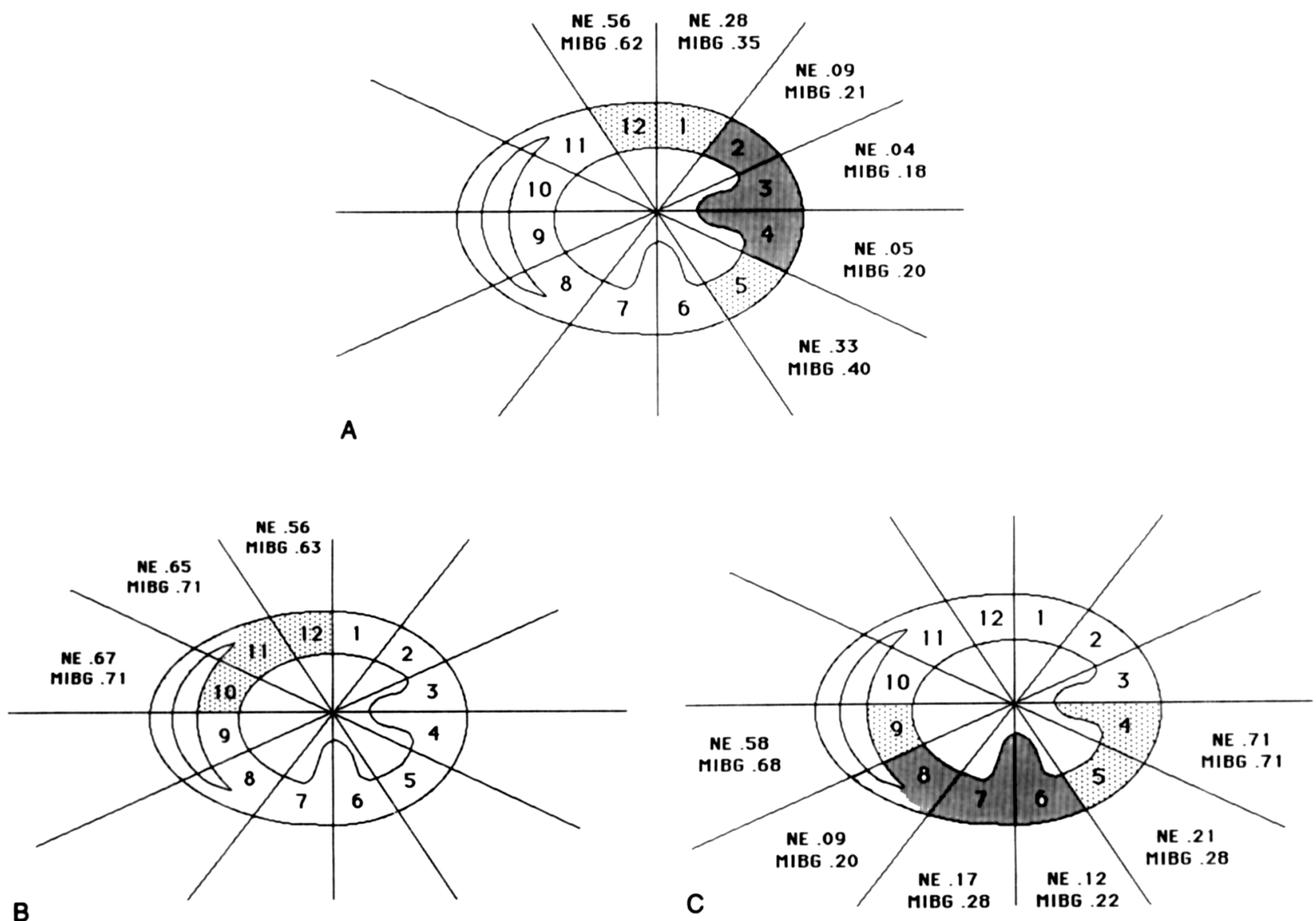

Fig. 1. Schematic drawings of basal slices showing the papillary muscles, anterior (prominence on the right) and posterior, and the radial segments of the left ventricle in three dogs. The relative diminutions in NE (markedly affected segments are darkly stippled and less affected areas are lightly stippled) and in ${ }^{125}$ I-MIBG concentrations are recorded. $A$, Heart from dog in which phenol treatment inscribed the standard $3 \times 2 \mathrm{~cm}$ rectangle on the anterior wall of left ventricle. (See Fig. 2, $B$ for scintigraphic portrayal of the same heart.) $B$, Heart from dog in which phenol treatment inscribed a smaller, $2 \times 2 \mathrm{~cm}$ rectangle on the anterior wall of left ventricle. The effects of phenol were more profound and extensive in the transverse slice $1 \mathrm{~cm}$ toward the apex in which only even-numbered radial segments were assayed for the relative ${ }^{125}$ I-MIBG content: segment No. 6, 0.79; segment No. 8, 0.31; segment No. 10, 0.36; and segment No. 12, 0.63. Apex samples were also affected: anterior apex NE, 0.09 and ${ }^{125}$ I-MIBG 0.24 ; posterior apex NE 0.37 and ${ }^{125}$ I-MIBG 0.40. (See Fig. 2, $C$ for scintigraphic portrayal of the same heart.) $C$, Heart from dog in which phenol treatment inscribed a $2 \times 2 \mathrm{~cm}$ rectangle on the inferior wall of the left ventricle. Abnormality appears as extensive as that produced when larger rectangles were inscribed. (See Fig. 2, $D$ for scintigraphic portrayal of same heart.)

ples of myocardium weighing 0.4 to $1.1 \mathrm{gm}$ were obtained from each of 12 radial segments of the basal slice of the left ventricle (Fig. 1), from the anterior and posterior aspects of the apex of the left ventricle, from the right ventricle at the same level as the basal slice of the left ventricle, and from the left and the right atria.

The samples were quickly frozen; then within 24 hours, they were placed in 9 volumes of iced $0.4 \mathrm{M}$ perchloric acid and homogenized by a Polytron homogenizer (Brinkmann Co., Westbury, N.Y.). Duplicate $0.5 \mathrm{ml}$ aliquots of whole homogenates were counted in a gamma counter. Window settings of the counter enabled selective detection of residual ${ }^{201} \mathrm{Tl}$ in the $120-180 \mathrm{keV}$ window, and then, by calculation, separation of ${ }^{125} \mathrm{l}-\mathrm{MIBG}$ from ${ }^{201} \mathrm{Tl}$ in the $15-80$
$\mathrm{keV}$ window. No residual ${ }^{123} \mathrm{I}-\mathrm{MIBG}$ could be detected. Possible metabolism of ${ }^{125}$ I-MIBG was assessed by determining the fraction of total ${ }^{125} I$ as free iodide with a Sep-Pak C-18 cartridge (Waters Associates, Milford, Mass. $)^{7,12}$ for at least five samples of each heart; the free iodide fraction was always less than $2 \%$ of the total ${ }^{125} \mathrm{I}$ in samples. The homogenates were centrifuged at $20,000 \times \mathrm{g}$ for 20 minutes at $5^{\circ} \mathrm{C}$, and the supernatants were analyzed for endogenous NE by high-performance liquid chromatography with a method ${ }^{7}$ adapted from a published technique. $^{3}$

Additional samples of myocardium were obtained from the even-numbered radial segments of slices obtained 1 $\mathrm{cm}$ above and $1 \mathrm{~cm}$ below the basal slice of left ventricle 
Table I. Concentrations of norepinephrine and ${ }^{125}$ I-MIBG in radial segments of left ventricle

\begin{tabular}{|c|c|c|c|}
\hline & $N E(n g / g m)$ & ${ }^{125} I-M I B G(n C i / g m) *$ & $M I B G / N E(n C i / \mu g)$ \\
\hline \multicolumn{4}{|l|}{ Sham-treated hearts (9) } \\
\hline Left ventricle: basal slice $\ddagger$ & $626 \pm 238 \S$ & $22.4 \pm 11.0$ & $38.1 \pm 13.6$ \\
\hline $\begin{array}{l}\text { Coefficient of variation for } 12 \text { radial } \\
\text { segments of each dog as percent }\end{array}$ & $\begin{array}{c}11.9 \pm 4.4 \\
(6.4-17.9) \dagger\end{array}$ & $\begin{array}{l}8.7 \pm 3.0 \\
(5.9-14.2)\end{array}$ & $\begin{array}{c}7.8 \pm 4.3 \\
(5.6-15.5)\end{array}$ \\
\hline \multicolumn{4}{|l|}{ Phenol-treated hearts (12) } \\
\hline $\begin{array}{l}\text { Left ventricle: basal slice, } \\
\text { unaffected segments } \ddagger\end{array}$ & $688 \pm 227$ & $26.5 \pm 8.7$ & $40.8 \pm 13.6$ \\
\hline
\end{tabular}

${ }^{*}$ Results adjusted to give a dose of $200 \mu \mathrm{Ci}$ of $125 \mathrm{I}-\mathrm{MIBG}$ to a $15 \mathrm{~kg}$ dog; tissues obtained 20 hours later.

+ Range in parentheses.

†For each heart, the mean of the radial segments from the basal slice was used in calculation; only unaffected radial segments were used for the phenol-treated hearts.

\&Mean \pm SD.

described above. These samples were assayed only for 125I-MIBG content to give added dimensions to the size of the region of neuron injury.

The basal slices of left ventricle were randomly selected from five dogs (three sham-treated and two phenoltreated) for incubation in a solution of $0.5 \%$ triphenyltetrazolium chloride for 15 minutes. This technique, ${ }^{14}$ along with measurement of residual ${ }^{201} \mathrm{Tl}$ in the samples, gave evidence of myocardial viability.

Statistical analysis. Coefficient of variation is the standard deviation expressed as a percent of the mean. This term is used to relate variability in data among the hearts of dogs to the variability of data found within a heart.

Within the sham-treated hearts, the mean concentrations of NE and of ${ }^{125}$ I-MIBG in apical segments were compared with the respective mean concentrations of $\mathrm{NE}$ and of ${ }^{125 I-M I B G}$ in the left ventricle by the two-tailed Student $t$ test in hypotheses that were independently developed. Similarly, the mean concentrations of NE and of ${ }^{125} \mathrm{I}-\mathrm{MIBG}$ in the atria were compared with respective values of the left ventricle. The Bonferroni inequalities correction $^{15}$ is made when segments could be considered in pairs, as for example from anterior and posterior apices, and from left and right atria.

By definition, the most affected segment in the phenoltreated hearts contained the lowest concentration of NE. The mean ${ }^{125}$ I-MIBG concentration in this most affected segment was compared with the mean ${ }^{125}$ I-MIBG in unaffected segments of the left ventricle by the two-tailed Student $t$ test.

Comparisons of respective segments in sham-treated and phenol-treated hearts were by the two-way analysis of variance.

\section{RESULTS}

Norepinephrine as an index of neuron disruption and correlation with ${ }^{125}$ I-MIBG. Table I records the concentrations of endogenous NE and ${ }^{125} \mathrm{I}-\mathrm{MIBG}$ in the radial segments of the basal slice of the left ventricle. Variability in both of these indices was moderate among the hearts from sham-treated dogs. However, within the left ventricle of a given dog, the variability from segment to segment in a the basal slice was relatively low: the coefficient of variation was always less than $18 \%$ for NE, and always less than $15 \%$ for ${ }^{125 I-M I B G}$. Therefore in spite of the moderate differences among dogs, within a heart there was reasonable uniformity in both NE and ${ }^{125}$ I-MIBG concentrations.

In addition, the levels of NE in the left ventricle varied among the hearts of the dogs in patterns that were not always the same as those developed from the concentrations of ${ }^{125}$ I-MIBG, and thus the ${ }^{125 I}$ MIBG/NE ratios in the left ventricle also exhibited moderate variability among dogs (Table I). Nevertheless, within a given dog's left ventricle, the ${ }^{125}$ I-MIBG/NE ratios were quite stable from segment to segment in the slice. Therefore when viewed as a pattern within a heart, the ${ }^{125} \mathrm{I}-\mathrm{MIBG} / \mathrm{NE}$ ratios in the left ventricle were also quite uniform.

In the phenol-treated hearts, the concentrations of NE in the radial segments of the basal slice of left ventricle were examined for abrupt changes. Although the maximum changes in NE concentrations in the regions of neuronal disruption were substantial and obvious, there were gradations in myocardial NE levels toward the edges of the disruption. Therefore, a radial segment was designated abnormal (and affected by phenol) if it contained $\mathrm{NE}$ at a concentration 3 standard deviations below the mean of what appeared to be a uniform NE concentration in "normal and unaffected" contiguous radial segments. These phenol-affected segments were always contiguous, and none had an NE concentration of more than $81 \%$ of the mean of the segments designated as unaffected. The unaffected segments in these phenol treated hearts had mean NE and ${ }^{125}$ I-MIBG concentrations and ${ }^{125} \mathrm{I}-\mathrm{MIBG} /$ 
Table II. Relative concentrations of norepinephrine and ${ }^{125}$ I-MIBG in samples of myocardium

\begin{tabular}{|c|c|c|c|}
\hline \multirow{2}{*}{$\begin{array}{l}\text { Sample of } \\
\text { myocardium }\end{array}$} & \multicolumn{3}{|c|}{$\begin{array}{c}\text { Ratios: Concentration-Mean concentration of unaffected radial } \\
\text { segments left ventricle }\end{array}$} \\
\hline & $N E$ & ${ }^{125} I-M I B G+$ & $M I B G / N E$ \\
\hline \multicolumn{4}{|l|}{ Phenol-treated hearts $(10)^{*}$} \\
\hline $\begin{array}{l}\text { Left ventricle: Most affected } \\
\text { radial segment }\end{array}$ & $0.064 \pm 0.036^{\mathrm{a}}$ & $0.339 \pm 0.131^{\mathrm{h}}$ & $7.35 \pm 5.78$ \\
\hline Left ventricle anterior apex & $0.119 \pm 0.070^{c}$ & $0.376 \pm 0.141^{\mathrm{c}}$ & $3.971 \pm 2.70$ \\
\hline Left ventricle posterior apex & $0.162 \pm 0.144^{\mathrm{c}}$ & $0.388 \pm 0.164^{\mathrm{c}}$ & $4.51 \pm 3.24$ \\
\hline Right ventricle & $0.886 \pm 0.324^{d}$ & $0.926 \pm 0.214^{\mathrm{d}}$ & $1.15 \pm 0.447$ \\
\hline Left atrium & $2.18 \pm 0.719^{d}$ & $1.57 \pm 0.408^{d}$ & $0.742 \pm 0.169$ \\
\hline Right atrium & $2.48 \pm 0.670^{\mathrm{d}}$ & $1.85 \pm 0.412^{\mathrm{d}}$ & $0.755 \pm 0.135$ \\
\hline \multicolumn{4}{|l|}{ Sham-treated hearts $(9)$} \\
\hline Left ventricle anterior apex & $0.681 \pm 0.118^{\circ}$ & $0.823 \pm 0.118^{e}$ & $1.22 \pm 0.208$ \\
\hline Left ventricle posterior apex & $0.629 \pm 0.194^{\mathrm{e}}$ & $0.770 \pm 0.154^{\mathrm{c}}$ & $1.29 \pm 0.358$ \\
\hline Right ventricle & $0.964 \pm 0.122^{e}$ & $0.950 \pm 0.136^{e}$ & $0.985 \pm 0.072$ \\
\hline Left atrium & $2.88 \pm 0.866^{f}$ & $1.94 \pm 0.487^{\mathrm{f}}$ & $0.709 \pm 0.219$ \\
\hline Right atrium & $2.73 \pm 0.797^{\mathfrak{f}}$ & $1.75 \pm 0.460^{x}$ & $0.664 \pm 0.163$ \\
\hline
\end{tabular}

Statistical analysis:

Values are mean \pm SD.

1. Comparing the mean of the most affected segment in left ventricle of phenol-treated hearts with mean of unaffected segments in left ventricle of phenol-treated hearts:

the NE concentration was low by definition.

$b_{p}<0.001$ by two-tailed Student's $t$ test.

2. Comparing segments of phenol-treated hearts with respective segments of sham-treated hearts by two-way analysis of variance: c $<0.001$.

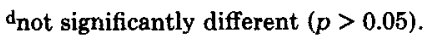

3. Comparing segments of sham-treated hearts with the mean of the respective left ventricle basal segments by two-tailed Student's $t$ test using Bonferroni inequalities correction ${ }^{15}$ :

enot significantly different $(p>0.05)$. $f_{p}<0.001$

4. Statistical comparisons were not made of MIBG/NE ratios.

*Radial segments from basal slice. In two dogs, phenol was applied to a smaller area of epicardium; results of hearts in these dogs were not included.

tSame segment as in NE calculation even though another segment may have contained slightly less ${ }^{125}$ I-MIBG (see text).

NE ratios comparable to those of radial segments from the sham-treated hearts (Table I).

When phenol was applied to inscribe the larger $(3 \times 2 \mathrm{~cm})$ rectangle on the epicardium, five to eight of the radial segments in the basal slice of left ventricle were affected according to the above criteria. One to four of the radial segments were considered markedly affected, containing less than $20 \%$ of the NE in the unaffected segments (Fig. 1 and Table II).

The concentrations of ${ }^{125}$ I-MIBG were reduced in samples to give a pattern of affected samples identical to that of NE (Fig. 1, $A$ and Table II). In the basic slice, the markedly affected segments (those containing less than $20 \%$ of the NE found in unaffected segments) were also those with lowest concentrations of ${ }^{225} \mathrm{I}-\mathrm{MIBG}$. The radial segment with the least NE (most affected) was the one containing the least ${ }^{125} \mathrm{I}-\mathrm{MIBG}$, except where the lowest NE and lowest ${ }^{125} \mathrm{I}-\mathrm{MIBG}$ were in adjacent segments that were almost equally affected.
Others ${ }^{16}$ have found the concentrations of NE to be lower in the apex than in the basal regions of the heart. Although the mean NE concentrations in the apical segments in the sham-treated hearts were lower than those in the basal slice, the differences were not significant (Table II). The levels of ${ }^{126} \mathrm{I}$ MIBG in these segments exhibited a similar pattern (Table II). However, in the apices of the phenoltreated hearts, both the NE and ${ }^{125 I-M I B G}$ concentrations were reduced even further, and compared with the respective apical NE and ${ }^{123}$ I-MIBG levels in the sham-treated hearts, the values in the phenoltreated hearts were significantly lower, $p<0.001$ for both NE and ${ }^{125}$ I-MIBG concentrations.

The respective mean levels of NE and ${ }^{125}$ I-MIBG in the right ventricle did not significantly differ in the two groups of dog hearts (Table II). Nevertheless, in three dogs, both NE and ${ }^{125}$ I-MIBG concentrations were more than three standard deviations below the reference mean of unaffected segments in the left ventricle. Probably the application of phenol 
reached part of the right ventricle in these three dogs.

In the multiple samples of myocardium from the regions of neuron disruption (designated by diminished concentrations of NE), the ${ }^{125}$ I-MIBG levels were also reduced, but these reductions were regularly less than those of NE. In the most affected samples of myocardium, residual ${ }^{125}$ I-MIBG levels averaged $34 \%$ of the mean of unaffected segments of left ventricle, while residual $\mathrm{NE}$ was $6.4 \%$ of the respective unaffected segment values (Table II). Concentrations of ${ }^{125}$ I-MIBG in segments from a slice above the standard basal slice tended to show lesser effects, while those from a slice below this basal region manifested similar or greater degrees of abnormality.

The atria were examined to include tissues unaffected by phenol. In the sham-treated hearts, the left and right atria contained significantly higher concentrations of $\mathrm{NE}$ values $(2.88$ and $2.73 \times$ respectively; each $p<0.001$ ) than those of the left ventricle (Table II), a phenomenon previously observed. ${ }^{17,18}$ However, concentrations of NE in the atria of the phenol-treated hearts did not differ significantly from those in sham-treated hearts. Similarly, the ${ }^{125}$ I-MIBG concentrations in both atria of sham-treated hearts were significantly higher than those in the respective left ventricle, but the values of ${ }^{125}$ I-MIBG in the sham-treated hearts were not different from those in the phenol-treated hearts (Table II). An additional phenomenon was noted. Although the ${ }^{125} \mathrm{I}-\mathrm{MIBG}$ concentrations in the atria were clearly higher than those in the left ventricle, the relative elevations of atrial ${ }^{125}$ I-MIBG were less than the relative increases in atrial NE.

In two dogs, phenol was applied to inscribe a rectangle of $2 \times 2 \mathrm{~cm}$ to determine if in vivo scintigraphy (see below) could detect smaller regions of adrenergic neuron disruption, regions that would include neuronal injury on the apex side of the area of the phenol application. Although the data from the hearts of those two dogs were not greatly different from those of other phenol-treated animals, they are not included in Table II. Such a restricted treatment with phenol on the anterior wall of the left ventricle produced smaller changes in concentrations of NE and of MIBG in the radial segments of the basal slice (Fig. 1, B), but the slice of left ventricle $1 \mathrm{~cm}$ toward the apex showed a higher concentration of MIBG (NE was not measured in inese segments). The apex of this heart was also affected. In the other dog, the effects of phenol on the inferior wall of the left ventricle appeared no less than those produced when phenol was applied to give a larger rectangle (Fig. 1, C). Nevertheless, the heart of this latter dog served as a model of neuron disruption in the inferior wall for scintigraphic evaluation.

Scintigraphy of ${ }^{123}$-MIBG. Variability in the concentration of ${ }^{123}$ I-MIBG within the walls of the left basal region of the left ventricle in sham-operated hearts was modest; in some dogs, the anterior wall on short-axis tomographs appeared to contain slightly less radioactivity than other myocardial walls. However, the radioactivity in the walls of the left ventricle was always continuous in all sham-treated hearts (Fig. 2, A). The effects of phenol treatment were readily distinguished as abnormal (Fig. $2, B$ ) and predicted faithfully the regions that would be identified as affected by ex vivo analysis of myocardial samples for NE and ${ }^{125}$ I-MIBG (Fig. 1, $A$ ). Not only was the region of phenol treatment abnormal, but the concentration of radioactivity was decreased on the apical side of the phenol-inscribed rectangle, including the apex of the the heart (Fig. 2,B), as was found in the excised samples from these regions.

When the region of phenol treatment was smaller, the abnormality in the anterior wall of the scintigraphic image was still easily detected (Fig. 2, C). Also, application of phenol to the inferior wall of the left ventricle resulted in images portraying diminished concentrations of ${ }^{123}$ I-MIBG in the same region (Fig. 2, $D$ ).

The images of ${ }^{123}$ I-MIBG in Fig. 2, $A$ through $D$ were created 18 to 20 hours after the injection of ${ }^{123} \mathrm{I}-\mathrm{MIBG}$, a time when a substantial fraction of the peak radioactivity (probably reached by 0.5 to 2 hours) had disappeared by biologic loss of MIBG, and physical decay of ${ }^{122}$ I (half-life, 13.3 hours). Scintigraphic images of hearts made 3 hours after the injection of ${ }^{123}$ I-MIBG were somewhat more distinct, probably because of the higher concentrations of radioactivity, but the configuration of and relative concentrations of the portrayed ${ }^{123}$ I were indistinguishable. Planar images of the heart obtained in the anterior view did not demonstrate the effects of phenol treatment as vividly.

Evidence for integrity of myocardium. Scintigraphic depictions of ${ }^{201} \mathrm{Tl}$ in the myocardium were identical in the sham-treated and phenol-treated hearts. The distribution of this radiopharmaceutical appeared uniform in the hearts of both groups of animals (Fig. 3). The residual ${ }^{201} \mathrm{Tl}$ (5 days after administration) in myocardium was uniformly distributed throughout the samples of left ventricle, and the concentrations of ${ }^{201} \mathrm{Tl}$ in the affected samples were the same as those in the unaffected radial segments.

The triphenyltetrazolium stain was uniform in all 


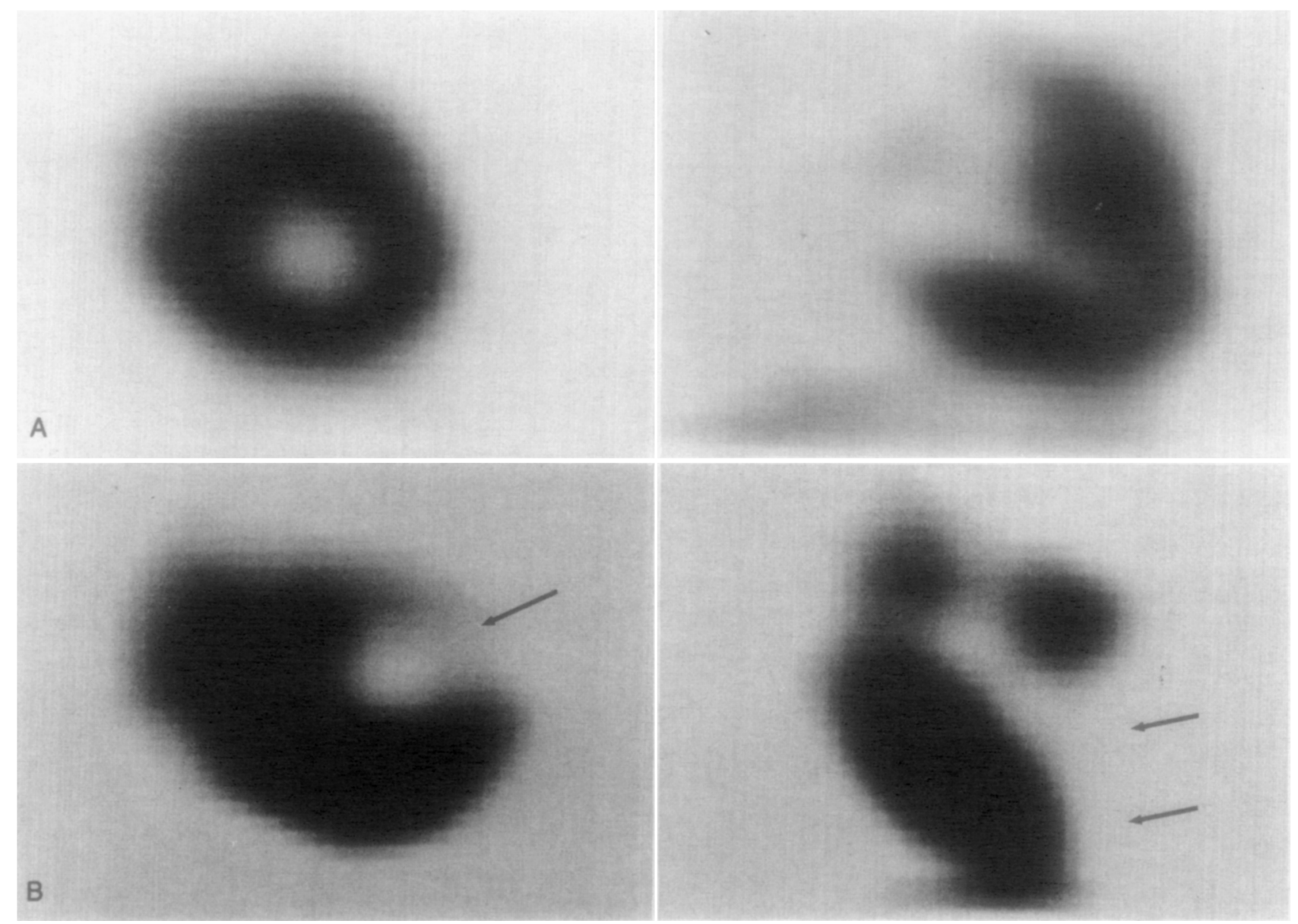

Fig. 2, A and B. Scintigraphy of dog's heart created 18 to 20 hours after injection of 5 mCi of ${ }^{123}$ I-MIBG. All images are tomographic sections of $6.5 \mathrm{~mm}$ thickness. A, Sham-treated heart. Left, Tomographic section through short axis in region where phenol was be applied to hearts of other dogs. Right, Vertical section midway through the heart; note that the radioactivity in apex is diminished compared to other regions. $B$, Phenol-treated heart; $3 \times 2 \mathrm{~cm}$ rectangle on the anterior wall of the left ventricle. Left, Section through short axis in region of phenol treatment; note absence of radioactivity in anterior wall region (arrow). This is the same projection of the heart schematically represented in Fig. 1, A. Right, Vertical section; note the absence of activity in the anterior wall giving an abnormality that extends into the apex (arrows).

hearts tested, giving no evidence for myocardial damage in the regions of phenol application.

Effects of stellectomy. Although excision of the left stellate ganglion interrupted the sympathetic modulation of left ventricular function, no regional or global changes in endogenous NE concentrations coud be detected in the hearts of seven dogs evaluated 5 to 7 days after stellectomy. There were also no regions in which levels of ${ }^{126}$ I-MIBG were diminished, so scintigraphy of these dogs was not performed.

\section{DISCUSSION}

The application of phenol to the epicardial surface of the heart is an established method of produc- ing tissue injury of only $0.25 \mathrm{~mm}$ in depth ${ }^{11}$ but sufficient to disrupt the adrenergic neurons that course through the epicardium toward their termination in the ventricular myocardium.,.$^{2,11}$ Vagal fibers apparently run a deeper course and are unaffected by the procedure. ${ }^{2,18}$ The adrenergic neurons are oriented from base to apex so that fibers on the apical side of an injury are also disrupted. ${ }^{2}$ Since NE is concentrated in the terminal fibers of adrenergic neurons, concentrations of this neurotransmitter within myocardium give evidence of neuron integrity..$^{16,20}$

In our experiments, the application of phenol to the epicardium of dogs' hearts regularly reduced the concentrations of $\mathrm{NE}$ in the myocardium in the 


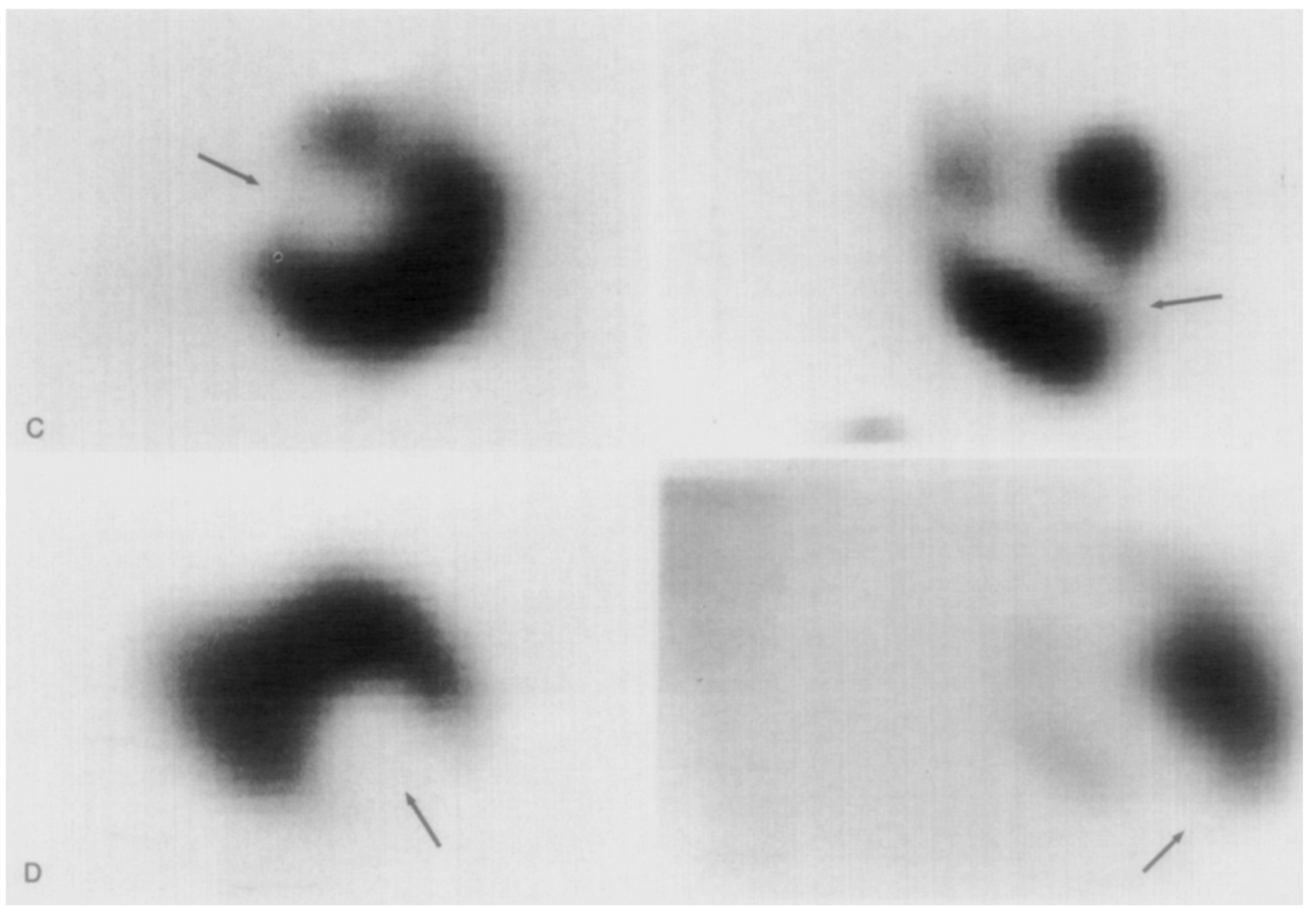

Fig. 2, C and D. Scintigraphy of dog's heart created 18 to 20 hours after injection of $5 \mathrm{mCi}$ of ${ }^{123} \mathrm{I}-\mathrm{MIBG}$. $C$, Phenol-treated heart; $2 \times 2 \mathrm{~cm}$ rectangle on the anterior wall of the left ventricle. Left, Section through short axis; diminished ${ }^{123} \mathrm{I}-\mathrm{MIBG}$ in anterior wall is easily seen (arrow). This is same projection of the heart schematically portrayed in Fig. 1, B. Right, Vertical section; region of low concentration of ${ }^{123}$ I-MIBG extends into the apex (arrow). $D$, Phenol-treated heart; $2 \times 2 \mathrm{~cm}$ rectangle on the inferior wall of left ventricle. (The area of the rectangle may have been larger.) Left, Section through short axis; note virtual absence of radioactivity in inferior wall (arrow). This is the same projection of the heart schematically portrayed in Fig. 1, C. Right, Vertical section; the region of diminished radioactivity in the inferior wall extends into the apex (arrow).

region of treatment and in the apex. The NE content declined to below $20 \%$ and often to below $10 \%$ of values of control samples of myocardium except in the heart of one dog, in which the NE concentration was less affected when the rectangle inscribed by phenol application was $2 \times 2 \mathrm{~cm}$ instead of $2 \times 3 \mathrm{~cm}$. (Even in this heart the injury to neurons was evident in the myocardium nearer the apex.)

We have previously shown ${ }^{7,8}$ that MIBG acts as an analog of NE and therefore regions of adrenergic neuron disruption should have reduced uptakes of radiolabeled MIBG. Others have reported in abstracts that reduced concentrations of myocardial ${ }^{123}$ I-MIBG, from diminished uptake ${ }^{21}$ or more rapid washout, ${ }^{22}$ reflect regions of neuron injury.

In the experiments described in this communica- tion, the concentrations of ${ }^{125}$ I-MIBG in myocardium were reduced in patterns similar to those of the NE. However, relatively higher concentrations of ${ }^{125}$ I-MIBG compared to those of NE were observed in the samples that were markedly affected by phenol treatment; the average difference was $28 \%$ (34 vs 6\%) between the radiopharmaceutical and the neurotransmitter in these samples. Possibly the higher ${ }^{125}$ I-MIBG residual in the region of injured adrenergic neurons relates to a non-neuronal component of the radiopharmaceutical in the myocardium. On the other hand, ${ }^{125}$ I-MIBG is probably taken up and concentrated in neurons as is injected ${ }^{3} \mathrm{H}$ $\mathrm{NE}$, and this uptake function differs from the synthesis of endogenous NE. Indeed, the neuronal uptake function has been shown to recover more 


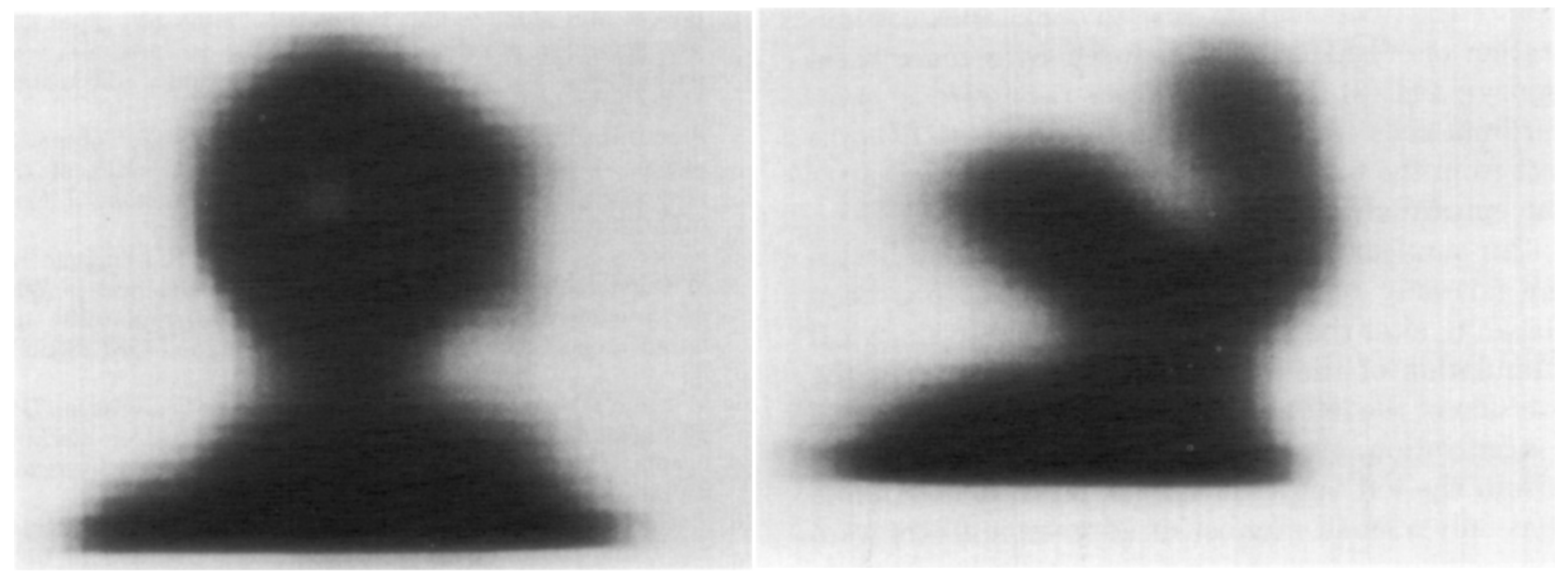

Fig. 3. Scintigraphy of phenol-treated dog heart created 15 minutes after injection of $2.5 \mathrm{mCi}$ of ${ }^{201} \mathrm{Tl}$. All images are tomographic sections and are comparable to those in Fig. 2. This is the same heart portrayed in Figs. 1, $A$ and $2, B$. Left, Section through short axis; ${ }^{201}$ Tl is uniformily distributed in left ventricle walls and image does not differ from those obtained in sham-treated hearts. Right, Vertical section; again ${ }^{201} \mathrm{Tl}$ distribution is normal.

quickly than does the synthesis of NE after neuronal injury. ${ }^{20}$ Thus the difference in relative concentrations of ${ }^{125}$ I-MIBG and NE may only indicate that different functions within adrenergic neurons were being tested. Nevertheless, the data support the concept that relative concentrations of ${ }^{125}$ I-MIBG, and therefore of any radiolabeled MIBG, are reliable indices of adrenergic neuron injury and integrity.

Since the application of phenol to ventricular epicardium reduced concentrations of ${ }^{125} \mathrm{I}-\mathrm{MIBG}$, both in the region of treatment and in the regions on the apex side of treatment, the in vivo distribution of ${ }^{123}$ I-MIBG should be similarly affected. In fact, the scintigraphic images of dog hearts demonstrated diminished ${ }^{123}$ I-MIBG in the predicted regions. This was true whether the phenol was applied to the anterior or the inferior walls of the left ventricle. Areas of phenol treatment as small as $2 \times 2 \mathrm{~cm}$ produced easily visualized abnormalities in the ${ }^{123}$ IMIBG scintigraphic images.

Previous experiments ${ }^{8}$ had suggested that the non-neuronal fraction of ${ }^{123} \mathrm{I}-\mathrm{MIBG}$ in the heart was higher at 2 to 3 hours than at 18 to 20 hours after injection; thus the latter time was chosen for acquisition of most data in the current studies. Nevertheless, the increment in the non-neuronal fraction of ${ }^{123}$ I-MIBG at 2 to 3 hours is probably small, and in any case the images at this time depicted the same abnormalities as seen at 18 to 20 hours. In addition, because more radioactivity was present in the heart at the earlier time, both the normal and the abnormal structures were then better resolved and appeared more distinct on the images. The heart cavity is always seen as a photopenic region, and therefore radioactivity in blood is not thought to contribute appreciably to measurements of the heart.

It is apparent that the distribution of ${ }^{125 I-M I B G}$ in the ex vivo analysis was not identical to that observed in images of the ${ }^{123}$ I-MIBG in vivo, particularly in the case of the dog hearts shown in Figs. 1, $B$ and 2, C. However, the discrepancies in the ex vivo and in vivo patterns are explained by the inherent limitations of dissecting the heart to include in its totality a small region of prior phenol application in the mid-basal slice that served as the reference for ex vivo analysis. Despite this impediment to the ex vivo reconstruction of phenol effects, the correlation between distributions of ${ }^{125}$ I-MIBG and ${ }^{123}$ I-MIBG appears to be well established by our data. Thus scintigraphy of ${ }^{123}$ I-MIBG will detect relatively small regions of adrenergic neuron disruption.

The patterns of ${ }^{123}$ I-MIBG concentrations within the heart should also be specific for the integrity of adrenergic neurons, whether or not there are associated abnormalities in the myocardium. The evidence indicates that the phenol injury was selective for the epicardium. In the phenol-treated hearts, ${ }^{201} \mathrm{Tl}$ was disseminated uniformily, and triphenyltetrazolium gave a normal staining pattern, demonstrating that the myocardium of these hearts was normal. Therefore, the disruption of adrenergic neurons must have occurred in the epicardium. The scintigraphic images of ${ }^{123}$ I-MIBG support these previously stated concepts ${ }^{211}$ : (1) adrenergic neurons course through the epicardium; (2) the direc- 
tion of the fibers is from base to apex (apex concentration of ${ }^{123}$ I-MIBG was reduced by a more basal injury); and (3) that the neurons penetrate to myocardium cells from the epicardium $\left({ }^{123}\right.$ I-MIBG was lost from the full thickness of myocardial wall after the epicardial injury).

Our surgical procedure to achieve a left stellectomy not only removed neural tissue but has been shown to alter the response of the heart to electrical stimulation of the left sympathetic chain. Yet the excision of the left stellate ganglion did not alter the concentration of endogenous NE and ${ }^{125}$ I-MIBG within the left ventricle 1 week later. It is possible that only a small number of adrenergic fibers were disrupted by the operation, too few to be detected by measurements of endogenous NE but sufficient to alter the normal response to electrical stimulation of the heart. The full effects of left stellectomy on the heart are in dispute. ${ }^{23,24}$ Nevertheless, we were unable to alter the NE concentrations, our index of adrenergic innervation, in regions of the heart by this type of procedure.

In summary, the relative distribution of radiolabeled MIBG in the heart is similar to that of endogenous NE. Disruptions of adrenergic neurons alter the levels of ${ }^{123}$ I-MIBG in myocardium, and these changes are portrayed on scintigraphic images. Of particular interest is the possibility that interdictions of adrenergic innervation in different regions of the heart-as would be caused, for example, by myocardial infarctions-may predispose to arrhythmias. Thus scintigraphic patterns of ${ }^{123}$ I-MIBG in the hearts of patients may correlate with, and be predictive of, arrhythmias.

We are indebted to Mrs. Annise L. Johnson for expert secretarial assistance and to Mr. Doug Heady for accurate assays of norepinephrine (NE). Mr. Chris Massin at the Phoenix Memorial Laboratory of the University of Michigan synthesized the radiolabeled MIBG.

\section{REFERENCES}

1. Braunwald E, Sonnenblick EH, Ross J Jr. Contraction of the normal heart. In: Braunwald E, ed. Heart disease. A textbook of cardiovascular medicine. Philadelphia: W.B. Saunders Company, 1984:437.

2. Martins JB, Zipes DP. Epicardial phenol interrupts refractory period responses to sympathetic but not vagal stimulation in canine left ventricular epicardium and endocardium. Circ Res 1980;47:33-40.

3. Yamada S, Yamamura HI, Roeske WR. Alterations in cardiac autonomic receptors following 6-hydroxydopamine treatment in rats. Mol Pharmacol 1980;18:185-92.

4. Graboys TB, Lower B, Podrid PJ, DeSilva R. Long-term survival of nationte with malignant vontricular arrhythmia treated with antiarrhythmic drugs. Am J Cardiol 1982; 50:437-43.

5. Esler M, Willette I, Leonard P, et al. Plasma noradrenaline kinetics in humans. J Auton Nerv Syst 1984;11:125-44.
6. Burber MJ, Mueller TM, Henry DP, Felten SY, Zipes DP. Transmural myocardial infarction in the dog produces sympathectomy in noninfarcted myocardium. Circulation 1983;67:787-96.

7. Sisson JC, Wieland DM, Sherman P, Manger TJ, Tobes MC, Jaques S. Meta-iodobenzylguanidine as an index of the adrenergic nervous system integrity and function. J Nucl Med 1987;28:1620-4.

8. Sisson JC, Shapiro B, Meyers L, Mallette S, Mangner TJ, Wieland DM, Glowniak JV, Sherman P, Beierwaltes WH. Meta-iodobenzylguanidine to map scintigraphically the adrenergic nervous system in man. I Nucl Med 1987;28:162536.

9. Wieland DM, Wu J-L, Brown LE, Manager TJ, Swanson DP, Beierwaltes WH. Radiolabeled adrenergic neuron-blocking agents: adrenomedullary imaging with [ $\left.{ }^{131} 1\right]$ Iodobenzyl-guanidine. J Nucl Med 1980;21:349-53.

10. Patterson E, Holland K, Eller BT, Lucchesi BR. Ventricular fibrillation resulting from ischemia at a site remote from previous myocardial infarction. A conscious canine model of sudden coronary death. Am J Cardiol 1982;50:1414-23.

11. Kaye MP, Brynjolfsson GG, Geis WP. Chemical epicardiectomy. Cardiologia 1968;53:139-49.

12. Mangner TJ, Tobes MC, Wieland DM, Sisson JC, Shapiro B. Metabolism of meta-I-131-iodobenzylguanidine in patients with metastic pheochromocytoma: concise communication. J Nucl Med 1986;27:37-44.

13. Goldstein DS, Feuerstein GZ, Izzo JL Jr, Kopin IJ, Keiser HR. Validity and reliability of liquid chromatography with electrochemical detection for measuring plasma levels of norepinephrine and epinephrine in man. Life Sci 1981;28:467-75.

14. Fishbein MC, Meerbaum S, Rit J, Lando U, Kanmatsuse K, Mercier JC, Corday E, Ganz W. Early phase acute myocardial infarct size quantitation: validation of the tripenyltetrazolium chloride tissue enzyme staining technique. AM HEART J 1981;101:593-600.

15. Snedecor GW, Cochran WG. Stastitical methods. 7th ed. Ames, The Iowa State University Press, Iowa: 1980:166.

16. Pierpont GL, DeMaster EG, Cohn JN. Regional differences in adrenergic function within the left ventricle. Am J Physiol 1984;246:H824-H829.

17. Angelakos ET. Regional distribution of catecholamines in the dog heart. Circ Res 1965;16:39-44.

18. Kaye MP, Priola DV, Coyle J. Depletion of ventricular catecholamine levels following peripulmonary neurectomy. Proc Soc Exp Biol Med 1970;135:844-8.

19. Barber MJ, Mueller TM, Davies BG, Zipes DP. Phenol topically applied to canine left ventricular epicardium interrupts sympathetic but not vagal afferents. Circ Res 1984;55:532-44.

20. Kaye MP, Tyce GM. Norepinephrine uptake as an indicator of cardiac reinnervation in dogs. Am $J$ Physiol 1978; 235:H289-H294.

21. Tuli M, Minardo J, Mock B, Werner R, Siddiqui A, Zipes D, Wellman $H$. SPECT with high purity 1-123-MIBG after transmural myocardial infarction (TMI), demonstrating sympathetic denervation followed by reinnervation in a dog model [Abstract]. J Nucl Med 1987;28:669.

22. Dae M, Botvinick E, O'Connell W, Huberty J, Herre J, Chin M, Hattner R. Regional myocardial MIBG washout parallels regional sympathetic innervation [Abstract]. J Nucl Med 1987;28:608.

23. Bhandrari Ak, Scheinman MM, Morady F, Svinarich J, Mason J, Winkle R. Efficacy of left cardiac sympathectomy in the treatment of patients with the long QT syndrome. Circulation 1987;70:1018-23.

24. Schwartz PJ, Zaza A. The rational basis and the clinical value of selective cardiac sympathetic denervation in the prevention of maligant arrhythmias. Eur Heart J 1986;7:107-18. 